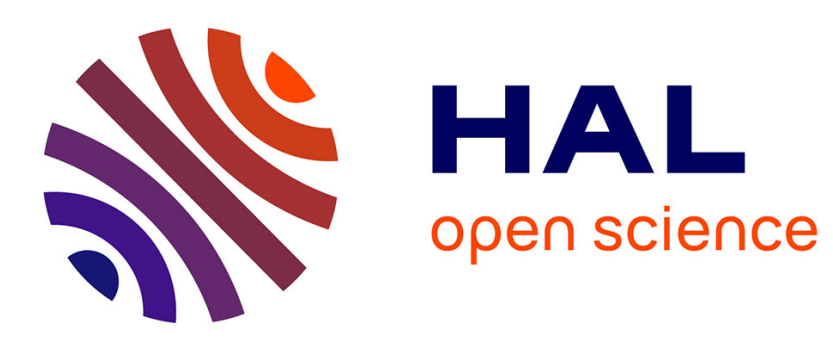

\title{
Parity and time-reversal breaking effects detected by resonant $\mathrm{x}$-ray scattering
}

\author{
Sergio Di Matteo, Y Joly
}

\section{To cite this version:}

Sergio Di Matteo, Y Joly. Parity and time-reversal breaking effects detected by resonant x-ray scattering. Physical Review B: Condensed Matter and Materials Physics (1998-2015), 2006, 74, 10.1103/physrevb.74.014403 . hal-03110643

\section{HAL Id: hal-03110643 \\ https://hal.science/hal-03110643}

Submitted on 14 Jan 2021

HAL is a multi-disciplinary open access archive for the deposit and dissemination of scientific research documents, whether they are published or not. The documents may come from teaching and research institutions in France or abroad, or from public or private research centers.
L'archive ouverte pluridisciplinaire HAL, est destinée au dépôt et à la diffusion de documents scientifiques de niveau recherche, publiés ou non, émanant des établissements d'enseignement et de recherche français ou étrangers, des laboratoires publics ou privés. 


\title{
Parity and time-reversal breaking effects detected by resonant x-ray scattering
}

\author{
S. Di Matteo ${ }^{1}$ and Y. Joly ${ }^{2}$ \\ ${ }^{1}$ Laboratori Nazionali di Frascati INFN, via E. Fermi 40, C.P. 13, I-00044 Frascati (Roma), Italy \\ ${ }^{2}$ Laboratoire de Cristallographie, CNRS, BP166, F-38042 Grenoble Cedex 9, France \\ (Received 21 March 2006; revised manuscript received 25 May 2006; published 6 July 2006)
}

\begin{abstract}
Recent experiments of x-ray spectroscopy have clearly shown that it is possible to get microscopic information on magnetic parity-breaking multipoles through these techniques. The aim of the present paper is to highlight some theoretical aspects related to the resonant x-ray scattering and analyze the main features of the signal in $\mathrm{GaFeO}_{3}$ through quantitative numerical calculations. Our results show that none of toroidal moments and octupoles as well as magnetic quadrupoles are negligible and that the signal depends on many specific crystallographic features of the system.
\end{abstract}

DOI: 10.1103/PhysRevB.74.014403

PACS number(s): 75.50.Gg, 78.70.Ck, 78.70.Dm

\section{INTRODUCTION}

The availability of second- and third-generation synchrotron radiation sources has opened new directions in the applications of resonant $\mathrm{x}$-ray scattering (RXS) to detect different kinds of order parameters in crystals. Nowadays, RXS is a very powerful and widespread technique to get information about charge, orbital, and magnetic degrees of freedom, ${ }^{1-3}$ due to the high sensitivity to ordered structures characteristic of x-ray diffraction, combined with the significant enhancement of the signal at energies close to an absorption edge. It is customary to express dispersion close to the edge as real and imaginary anomalous scattering terms $f^{\prime}+i f^{\prime \prime}$, related by Kramers-Kronig transform, to be added to the Thomson atomic scattering factor $f_{0}$, in order to get the total $\mathrm{x}$-ray amplitude as $f=f_{0}+f^{\prime}+i f^{\prime \prime}$ [see Eq. (1)]. Up to the beginning of the 1980s, it was usually assumed that $f^{\prime}$ and $f^{\prime \prime}$ were real quantities, with a simple, scalar, polarization dependence: however, after the seminal papers by Templeton and Templeton ${ }^{4}$ and Dmitrienko, ${ }^{5}$ nowadays we know that many effects can be singled out by the tensor character of the polarization and wave-vector dependence, and this is at the origin of the success of this technique to detect orbital and charge orderings, as well as structural anisotropies. Moreover, it became clear that $f^{\prime}$ and $f^{\prime \prime}$ were not even real quantities, depending on the behavior of the transition matrix elements included in their definitions, as shown, e.g., in Eqs. (2) and (3). For example, in the dipole-dipole (E1-E1) approximation both $f^{\prime}$ and $f^{\prime \prime}$ can be complex, due to an imaginary contribution coming from the transition matrix elements (3). As detailed below, all these features of the x-ray amplitude allow one to select, through an intricated interplay of interference effects, the physical variables of interest.

While the theory and phenomenology of magnetic resonant diffraction in $E 1-E 1$ conditions are very well studied, ${ }^{6}$ the possibility to detect small effects related to magnetic parity-breaking signals with RXS is still at its infancy. The latter, in fact, would require a way to select just magnetic terms in the dipole-quadrupole (E1-E2) channel, which are small compared to nonmagnetic terms due to the $\frac{\hbar \omega}{m c^{2}}$ factor ( $\hbar \omega$ is the energy of the incoming photon and $m c^{2}$ $=511 \mathrm{keV}$ the electron rest energy) and are further depressed compared to magnetic $E 1-E 1$ terms because of the radial $E 2$ matrix element. Therefore, some tricks are required in order to measure this signal: for example, a theoretical suggestion ${ }^{7}$ to reveal the ordered antiferromagnetic arrangement of toroidal moments in $\mathrm{Li}_{2} \mathrm{VOSiO}_{4}$ has been recently advanced, for a class of reflections where any other multipolar contribution is forbidden. Still more clever appears, however, the recent experiment performed on $\mathrm{GaFeO}_{3}$ by Arima et al., ${ }^{8}$ who found a beautiful way to take full advantage of the interference properties intrinsic in RXS. In fact, noticing that the magnetic anomalous amplitude in the E1-E2 channel is in phase with the nonmagnetic $E 1-E 1$ and Thomson parts, they could measure the existence of the magnetic, parity-breaking E1-E2 terms, mainly associated with toroidal moments, through their reciprocal interference: we shall call this technique MEXS (magnetoelectric x-ray scattering), after them.

The interest in measuring toroidal moments with RXS stems from the fact that they represent a good indicator of correlations between magnetism and ferroelectricity ${ }^{9}$ and they could even play a role in the physics of magnetic interfaces, since the inversion symmetry is broken at interfaces. Moreover, resonant techniques do in general allow microscopic information that cannot be reached through the usual macroscopic measurements like the magnetoelectric susceptibility. Indeed, while the latter is related to bulk properties, RXS spectra give direct information on the density of states projected on the resonant ion, both in energy space and in real space, through the azimuthal dependence.

The aim of this paper is threefold: first, we want to put the achievements of Ref. 8 on a more quantitative basis, by means of a series of numerical $a b$ initio calculations with the FDMNES program, ${ }^{10}$ in order to confirm the physical origin of the detected signal also through a different method than in Ref. 8. In the second place we show, both analytically and numerically, some subtleties inherent with the interference technique and apparently overlooked by the authors of Ref. 8. In particular, detailed calculations show that the signal strongly depends on the iron filling at both Fe sites and $\mathrm{Ga}$ sites. Here Fe sites are the sites nominally occupied by Fe atoms and $\mathrm{Ga}$ sites those nominally occupied by $\mathrm{Ga}$ atoms: indeed, Fe ions have a finite probability to occupy Ga sites, as shown in Ref. 11. Actually, the inversion of the signal at $(0 \overline{4} 0)$ compared to $(040)$ is strongly related to this aspect. In principle, this characteristic could be used to confirm the 
filling values obtained from the neutron scattering, ${ }^{11}$ even though the sensitivity of this technique to the Fe partial occupancy of Ga sites is not so developed as with neutron powder diffraction. Finally, after demonstrating that MEXS has indeed detected magnetic, parity-breaking quantities, we focus on the possibility to single out the toroidal contribution and the one arising from the magnetic quadrupole, ${ }^{7,9,12}$ on the basis of the polarization and wave-vector dependence of the signal. We believe that all these characteristics offer a good example to show the precise role of interference between anomalous and Thomson parts, and therefore reveal all the opportunities that can be covered by this technique, as well as the necessity to perform detailed quantitative calculations in order to correctly interpret the experimental data.

\section{THOMSON-ANOMALOUS INTERFERENCE IN RXS: THE CASE OF $\mathrm{GaFeO}_{3}$}

In RXS the global process of photon absorption, virtual photoelectron excitation, and photon reemission is coherent throughout the crystal, thus giving rise to the usual Bragg diffraction condition

$$
F=\sum_{j} e^{i Q \cdot \vec{Q}_{j}}\left(f_{0}(j)+f_{j}^{\prime}+i f_{j}^{\prime \prime}\right) .
$$

Here $\vec{R}_{j}$ stands for the position of the scattering ion $j, \vec{Q}$ is the diffraction vector, and $f_{0}(j)$ is the Thomson factor of the $j$ th atom. The resonant part $f_{j}^{\prime}+i f_{j}^{\prime \prime}$ is the anomalous atomic scattering factor, given, at resonance where $\hbar \omega \simeq\left(E_{n}-E_{g}\right)$, by the expression ${ }^{13}$

$$
f_{j}^{\prime}-i f_{j}^{\prime \prime} \equiv f_{j}(\omega)=m_{e} \omega^{2} \sum_{n} \frac{M_{n g}^{o^{*}}(j) M_{n g}^{i}(j)}{\hbar \omega-\left(E_{n}-E_{g}\right)+i \Gamma_{n} / 2},
$$

where $\hbar \omega$ is the photon energy, $m_{e}$ the electron mass, $E_{g}$ the ground-state energy, and $E_{n}$ and $\Gamma_{n}$ are the energy and inverse lifetime of the excited states. ${ }^{14}$ The sum is extended over all the excited states of the system. In Eq. (2), $f_{j}^{\prime}$ and $f_{j}^{\prime \prime}$ can be identified with the real and imaginary parts as determined by the resonant denominator. Moreover, we introduced the transition matrix elements of matter-radiation interaction in the $\mathrm{x}$-ray regime:

$$
M_{n g}^{i(o)}(j)=\left\langle\psi_{n}\left|\hat{O}^{i(o)}\right| \psi_{g}(j)\right\rangle,
$$

where the operator $\hat{O}$ is written through the multipolar expansion of the photon field up to electric dipole $(E 1)$ and quadrupole $(E 2)$ terms: ${ }^{15}$

$$
\hat{O}^{i(o)}=\vec{\epsilon}^{i(o)} \cdot \vec{r}\left(1+\frac{1}{2} i \vec{q}^{i(o)} \cdot \vec{r}\right) .
$$

In Eq. (3), $\psi_{g}(j)$ is the core ground state centered around the $j$ th atom and $\psi_{n}$ the photoexcited state, whereas in Eq. (4), $\vec{r}$ is the electron position measured from the absorbing ion, $\vec{\epsilon}^{i(o)}$ is the polarization of the incoming (outgoing) photon, and $\vec{q}^{i(o)}$ is its corresponding wave vector. In general the matrix element (3) can be complex, and therefore there is no advantage in considering $f_{j}^{\prime}$ and $f_{j}^{\prime \prime}$ separately, and in what

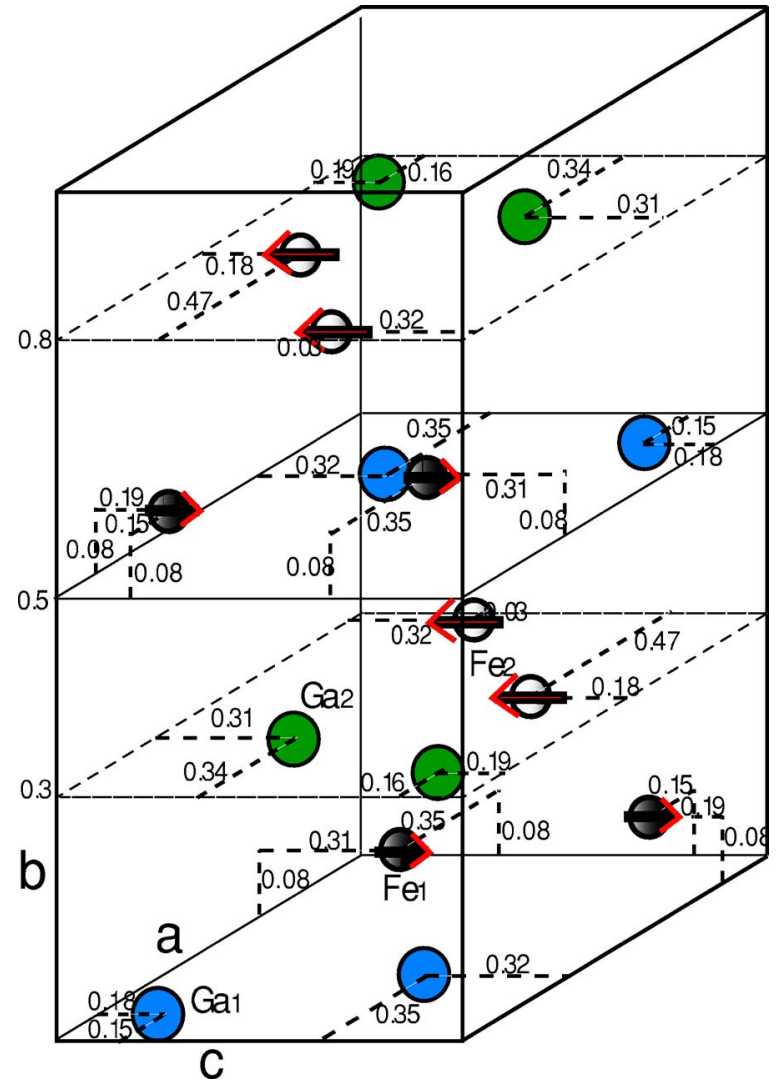

FIG. 1. (Color online) Structure of $\mathrm{GaFeO}_{3}$ : magnetic moments are shown along the crystallographic $c$ axis. Electric polarizations are along the $b$ axis. Toroidal moments are directed along the $a$ axis. Oxygen atoms, not shown, form strongly distorted octahedra around $\mathrm{Ga}_{2}$ and $\mathrm{Fe}$ ions and strongly distorted tetrahedra around $\mathrm{Ga}_{1}$ ions.

follows we shall deal mainly with $f_{j}$ (except for the analysis of Appendix B, where $f^{\prime}$ and $f^{\prime \prime}$ are used as they are known to be real).

In the case of the RXS interference technique, the anomalous scattering amplitude interferes with the Thomson factor of all other charge distributions: at the $\mathrm{Fe} K$ edge in $\mathrm{GaFeO}_{3}$, for example, the Fe resonant signal interferes not only with Fe Thomson $f_{0}$, but also with those of $\mathrm{Ga}$ and $\mathrm{O}$, and the latter turn out to be not negligible, as we shall see below.

\section{The case of $\mathrm{GaFeO}_{3}$}

The system under consideration, $\mathrm{GaFeO}_{3}$, is an orthorhombic piezoelectric crystal which also shows a spontaneous magnetization, below about $T_{c} \simeq 200 \mathrm{~K} .{ }^{16}$ Its magnetic Shubnikov group is $m^{\prime} 2^{\prime} m$. In the unit cell there are two groups of inequivalent $\mathrm{Fe}$ atoms, two groups of inequivalent $\mathrm{Ga}$ atoms, and six groups of inequivalent $\mathrm{O}$ atoms, each with $4 a$ Wyckoff number and no local symmetries (see Fig. 1). We have chosen, as in previous works about this system, ${ }^{11,17}$ the space group $P c 2_{1} n$, rotated with respect to the No. 33 of the International Tables for Crystallography ${ }^{18}\left(P_{n a 2_{1}}\right)$, in such a way that the magnetization lies along the $c$ axis and electric polarization is along $b$. The coordinates of the equivalent positions are, for all $\mathrm{Fe}, \mathrm{Ga}, \mathrm{O}$ atoms, $s_{1}$ 
TABLE I. Coordinates $(x, y, z)$ of the different $\mathrm{Fe}, \mathrm{Ga}$, and $\mathrm{O}$ atoms and probability $(p)$ to get an iron atom in the corresponding site.

\begin{tabular}{lcccc}
\hline \hline & $x$ & $y$ & $z$ & $p$ \\
\hline $\mathrm{Fe}_{1}$ & 0.1538 & 0.5831 & 0.1886 & 0.77 \\
$\mathrm{Fe}_{2}$ & 0.0346 & 0.7998 & 0.6795 & 0.70 \\
$\mathrm{Ga}_{1}$ & 0.1500 & 0.0000 & 0.1781 & 0.18 \\
$\mathrm{Ga}_{2}$ & 0.1593 & 0.3073 & 0.8106 & 0.35 \\
$\mathrm{O}_{1}$ & 0.3228 & 0.4262 & 0.9716 & 0 \\
$\mathrm{O}_{2}$ & 0.4864 & 0.4311 & 0.5142 & 0 \\
$\mathrm{O}_{3}$ & 0.9979 & 0.2022 & 0.6541 & 0 \\
$\mathrm{O}_{4}$ & 0.1593 & 0.1974 & 0.1480 & 0 \\
$\mathrm{O}_{5}$ & 0.1695 & 0.6717 & 0.8437 & 0 \\
$\mathrm{O}_{6}$ & 0.1736 & 0.9383 & 0.5166 & 0 \\
\hline \hline
\end{tabular}

$\equiv(x, y, z), \quad s_{2} \equiv(-x, y+1 / 2,-z), \quad s_{3} \equiv(x+1 / 2, y+1 / 2,1 / 2$ $-z)$, and $s_{4} \equiv(1 / 2-x, y, 1 / 2+z)$. The corresponding values are given in Ref. 11, and we report them in Table I for clarity because these values are used in our numerical calculations.

By taking into account the magnetic structure, equivalent sites $s_{1}, s_{2}, s_{3}$, and $s_{4}$ can be related to the first site $a$ by the following symmetry operations, respectively: $\left(\hat{E}, \hat{T} \hat{C}_{2 b}, \hat{m}_{c}, \hat{T} \hat{m}_{a}\right)$, where $\hat{E}$ is the identity, $\hat{T}$ is the timereversal operator, $\hat{C}_{2 b}$ is the $\pi$-rotation operator around $b$ axis, and $\hat{m}_{a}\left(\hat{m}_{c}\right)$ is the mirror plane orthogonal to the $a$ axis (c axis).

Therefore, the anomalous scattering amplitude at the Fe $K$ edge for the $(0 k 0)$ reflection, when summed over all equivalent sites, becomes

$$
F_{j}=\left(1+\hat{T} \hat{m}_{a}\right)\left(1+(-)^{k} \hat{m}_{c}\right) f_{j} .
$$

Here $f_{j}$ is given by Eq. (2) and $F_{j}$ is the global anomalous scattering amplitude of the four equivalent atoms $s_{1}, s_{2}, s_{3}$, and $s_{4}$ : notice that both depend on $k$ at the E1-E2 level, but not at the $E 1-E 1$, as clear from Eq. (4). The wave-vector dependence for time-reversal even and odd terms in the E1$E 2$ channel is detailed in Appendix A. The index $j$ runs over the four possible inequivalent sites having a finite probability of hosting a $\mathrm{Fe}$ ion (i.e., $\mathrm{Fe}_{1}, \mathrm{Fe}_{2}, \mathrm{Ga}_{1}$, and $\mathrm{Ga}_{2}$ ). In fact, as determined by neutron diffraction refinement, ${ }^{11} \mathrm{Fe}$ ions have a non-negligible probability to be found at sites nominally taken by $\mathrm{Ga}$ ions-i.e., $\mathrm{Ga}_{1}$ and $\mathrm{Ga}_{2}$.

The symmetry operations in Eq. (5) act only on the polarization-wave-vector tensorial part of $f_{j}$, as detailed, e.g., in Ref. 19. In order to get the total anomalous scattering amplitude at the Fe $K$ edge for the $(0 k 0)$ reflection, we need to sum up the $F_{j}$ over the four unequivalent sites where $\mathrm{Fe}$ ions have probability to reside, weighted by such a probability and by the corresponding Bragg phases:

$$
f_{a}=\sum_{j=1}^{4} p_{j} e^{2 \pi i k y_{j} F_{j}}
$$

Here $y_{j}$ is the $y$ coordinate of the unequivalent site $j$ (i.e., $y_{1}=0.5831, y_{2}=0.7998, y_{3}=0.0000$, and $\left.y_{4}=0.3073\right), F_{j}$ its
TABLE II. Relative contribution of $\mathrm{Fe}, \mathrm{Ga}$, and $\mathrm{O}$ Thomson and anomalous factors to the interference at the Fe pre- $K$ edge. $u$ and $v$ are define in Appendix B.

\begin{tabular}{lcc}
\hline \hline & $u+i v$ & $f^{\prime}+i f^{\prime \prime}$ \\
\hline $\mathrm{Fe}(040)$ & $-14.2+i 143$ & $-6.2+i 1.1$ \\
$\mathrm{Fe}(0 \overline{4} 0)$ & $-14.2-i 143$ & $-6.4+i 1.2$ \\
$\mathrm{Ga}(040)$ & $111+i 96.9$ & $-8.6+i 0.2$ \\
$\mathrm{Ga}(0 \overline{4} 0)$ & $111-i 96.9$ & $-0.9+i 8.52$ \\
$\mathrm{O}(040)$ & $-4.3-i 125$ & $0.9-i 1.3$ \\
$\mathrm{O}(0 \overline{4} 0)$ & $-4.3+i 125$ & $-1.0+i 1.3$ \\
\hline \hline
\end{tabular}

corresponding atomic scattering factor summed over equivalent sites [Eq. (5)], and $p_{j}$ is the probability to have an iron atom at site $j$, with the constraint $\sum_{j=1}^{4} p_{j}=2$ because there are globally two Fe ions per unit cell. For example, the nominal probability is $p_{1}=p_{2}=1$ and $p_{3}=p_{4}=0$, while the measured probabilities, as given in Ref. 11 , are $p_{1}=0.77, p_{2}=0.70, p_{3}$ $=0.18$, and $p_{4}=0.35$. The marked asymmetry of $\mathrm{Ga}_{1}$ sites probably comes from the fact that the latter one has an almost tetrahedral oxygen environment, contrary to the other three sites, which have a distorted octahedral environment. The Thomson scattering amplitude from $\mathrm{Ga}$ and $\mathrm{Fe}$ atoms, when summed over the whole cell for $k=$ even, is given by the complex number

$$
f_{0 k}=4 \sum_{j=1}^{4}\left[p_{j} f_{a t}^{\mathrm{Fe}}+\left(1-p_{j}\right) f_{a t}^{\mathrm{Ga}}\right] e^{2 \pi i k y_{j}},
$$

where $f_{a t}^{\mathrm{Fe}}$ and $f_{\text {at }}^{\mathrm{Ga}}$ are iron and gallium Thomson scattering factors, respectively, the factor of 4 counts the number of equivalent atoms (they all sum up for $k$ even), and $\left(1-p_{j}\right)$ is the probability that the $j$ th site will be occupied by a Ga atom. The $k$ dependence is explicitly shown in the label. Similarly the Thomson scattering of oxygens reads

$$
f_{0 k}^{\mathrm{O}}=4 \sum_{j=1}^{6} f_{a t}^{\mathrm{O}} e^{2 \pi i k \tilde{y}_{j}},
$$

where we took into account the fact that there are six $\tilde{y}_{j}$ inequivalent oxygen positions. In Eqs. (7) and (8), even though not explicitly written, we have inserted as well the $\mathrm{Ga}$ and $\mathrm{O}$ anomalous scattering contributions at the Fe $K$ edge, respectively: however, a posteriori, we found that their weight is negligible compared to $f_{a t}^{\mathrm{Ga}}$ and $f_{a t}^{\mathrm{O}}$, as shown in Table II.

\section{RESULTS AND DISCUSSION}

The experiment performed in Ref. 8 was structured as follows: an external magnetic field was applied along the easy axis $c$ while measuring the $(0 k 0)$ intensity and then reversed. The two signals were subsequently subtracted in such a way as to single out just the interference term involving the magnetic term of $E 1-E 2$ origin. It is important to stress here that the experiment was performed in the unrotated $\sigma \sigma$ channel-i.e., when both incoming and outgoing 
polarizations are orthogonal to the scattering plane-in such a way that no magnetic amplitude is allowed from the anomalous $f_{a}$ term in the $E 1-E 1$ approximation. Moreover, for $k=4 n$, with $n$ any integer, there is an approximated extinction rule for nonresonant magnetic scattering, differently from the case $k=4 n+2$. The expected intensities at (040) in the two opposite directions of the applied magnetic field $\left(I^{ \pm}\right)$ are

$$
\begin{aligned}
& I^{+}(040)-I^{-}(040) \\
& \quad \equiv d I=\left|f_{0 k}+f_{0 k}^{O}+f_{a}^{n m}+f_{a}^{m}\right|^{2}-\left|f_{0 k}+f_{0 k}^{O}+f_{a}^{n m}-f_{a}^{m}\right|^{2} .
\end{aligned}
$$

In the case of the $(0 \overline{4} 0)$ reflection we have, instead,

$$
\begin{aligned}
& I^{+}(0 \overline{4} 0)-I^{-}(0 \overline{4} 0) \\
& \quad \equiv d \tilde{I}=\left|f_{0 \bar{k}}+f_{0 \bar{k}}^{O}+\tilde{f}_{a}^{n m}+\tilde{f}_{a}^{m}\right|^{2}-\left|f_{0 \bar{k}}+f_{0 \bar{k}}^{O}+\tilde{f}_{a}^{n m}-\tilde{f}_{a}^{m}\right|^{2} .
\end{aligned}
$$

In Eqs. (9) and (10) we have introduced the magnetic and nonmagnetic parts of the anomalous term $f_{a}$ at the Fe $K$ edge as $f_{a}=f_{a}^{n m}+f_{a}^{m}$, which are, respectively, even and odd under time reversal (i.e., reversal of the magnetic direction). Moreover, we defined

$$
\tilde{f}_{a}^{m} \equiv \sum_{j=1}^{4} p_{j} e^{-2 \pi i k y_{j}} \tilde{F}_{j}=-\sum_{j=1}^{4} p_{j} e^{-2 \pi i k y_{j}} F_{j}
$$

due to the fact that when $\vec{Q} \rightarrow-\vec{Q}$, the $E 1-E 2$ anomalous term $F_{j}$ changes sign, being odd with respect to $\vec{Q}$, as shown in Appendix A. Notice, however, that $\tilde{f}_{a}^{m} \neq-f_{a}^{m}$, due to the Bragg factor $e^{-2 \pi i k y_{j}}$. We can rewrite Eqs. (9) and (10) as, respectively, ${ }^{20}$

$$
d I=4 \operatorname{Re}\left[\left(f_{0 k}+f_{0 k}^{O}+f_{a}^{n m}\right)^{*} f_{a}^{m}\right]
$$

and

$$
d \widetilde{I}=4 \operatorname{Re}\left[\left(f_{0 \bar{k}}+f_{0 \bar{k}}^{O}+\tilde{f}_{a}^{n m}\right)^{*} \tilde{f}_{a}^{m}\right] .
$$

At this point, it is worthwhile to linger on the explicit numerical calculation for $d I$ and $d \tilde{I}$ performed through the FDMNES program in the magnetic multiple-scattering mode and draw some comments in the light of the experimental results of Ref. 8, to check that we can first of all grasp the correct order of magnitude of the signal. The calculated spectra are shown in Fig. 2, together with the (020) reflection, where the E1-E2 magnetic signal is superimposed onto a magnetic nonresonant background. The absorption curve is also drawn, as a reference for the energy. Both (040) and $(0 \overline{4} 0)$ show a qualitative behavior well in keeping with the experimental data, with a pronounced bump at the Fe pre- $K$ edge, whose relative intensity $d I / I$ is some units in $10^{-4}$, like the experimental feature. Notice that we have a better agreement with the experiment when we suppose that the Fe ion at $\mathrm{Ga}_{1}$ site is not magnetic, again indicating that tetrahedral sites behave differently from octahedral ones.

Before commenting further on the characteristics of the signal evaluated numerically, it is possible to show analyti-

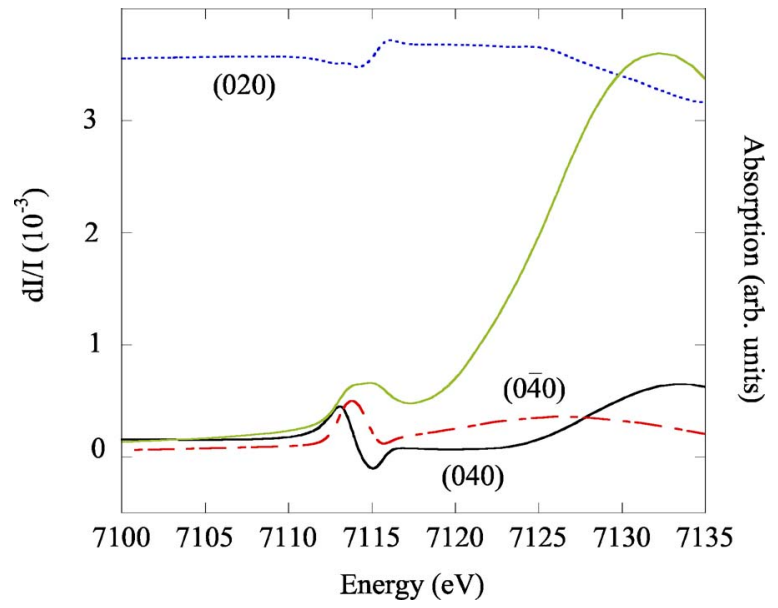

FIG. 2. (Color online) MEXS signal for the actual filling value. The absorption curve is drawn as a reference for the energy.

cally that $d I$ and $d \widetilde{I}$ are not necessarily opposite. By expressing the real and imaginary parts of the Thomson Bragg terms as $u$ and $v$ and as $\tilde{u}$ and $\widetilde{v}$ those of the anomalous Bragg terms (see Appendix B for definitions and calculations), we get the following expression:

$$
\begin{aligned}
d I+d \widetilde{I}= & 2 f_{m}^{\prime \prime}(k)\left\{\widetilde{u}(k, p)\left[v(k, p)+f_{n m s}^{\prime}\right]\right. \\
& \left.-\widetilde{v}(k, p)\left[u(k, p)+f_{n m c}^{\prime}\right]\right\} \\
& +2 f_{m}^{\prime}(k)\left[\widetilde{u}(k, p) f_{n m s}^{\prime \prime}-\widetilde{v}(k, p) f_{n m c}^{\prime \prime}\right],
\end{aligned}
$$

from which it is clear that what makes $d I+d \tilde{I} \neq 0$ is the sum of two contributions: on the one side, the interference between $f_{m}^{\prime \prime}(k)$ and the $p$-dependent Thomson terms $u$ and $v$ and on the other, the interference of $f_{m}^{\prime}(k)$ and $f_{m}^{\prime \prime}(k)$ with, respectively, the Templeton parts of $f_{n m}^{\prime \prime}$ and $f_{n m}^{\prime}$ in the E1-E1 contribution, called here $f_{n m c}$ and $f_{n m s}$, as detailed in Appendix B. It is the latter term which is responsible, for example, for the differences between the $(040)$ and $(0 \overline{4} 0)$ reflections shown as a solid line in Fig. 3. Here we show the signal coming from a single site $\mathrm{Fe}_{1}$, with $p_{1}=1$, for both the total intensity and the one coming from the simple interference of $f_{a}^{m}$ with $f_{a t}^{\mathrm{Fe}}$ : in the latter case, the relation $d I+d \widetilde{I}=0$ is obtained exactly, as expected from the theoretical analysis above.

The dependence of the signal on the filling probabilities $p_{j}$ is clear from Fig. 4, where it is shown how the spectra vary in case of "nominal" filling $\left(p_{1}=p_{2}=1\right.$ and $\left.p_{3}=p_{4}=0\right)$ and of the real one $\left(p_{1}=0.77, p_{2}=0.70, p_{3}=0.18\right.$, and $\left.p_{4}=0.35\right)$.

It should be noticed that the calculated nonresonant signal at the (020) reflection is about twice as big as the experimental one. In fact, as can be deduced from Fig. 2, the ratio of this latter with the resonant peak at (040) is about 8, while the experimental ratio is about 4 (see Ref. 8). As the relative intensity $d I / I$ is well reproduced by our calculation, we believe that the origin of the discrepancy lies in the evaluation of the nonresonant magnetic scattering with the extrapolation at low energies of the high-energy expression of Ref. 22. 


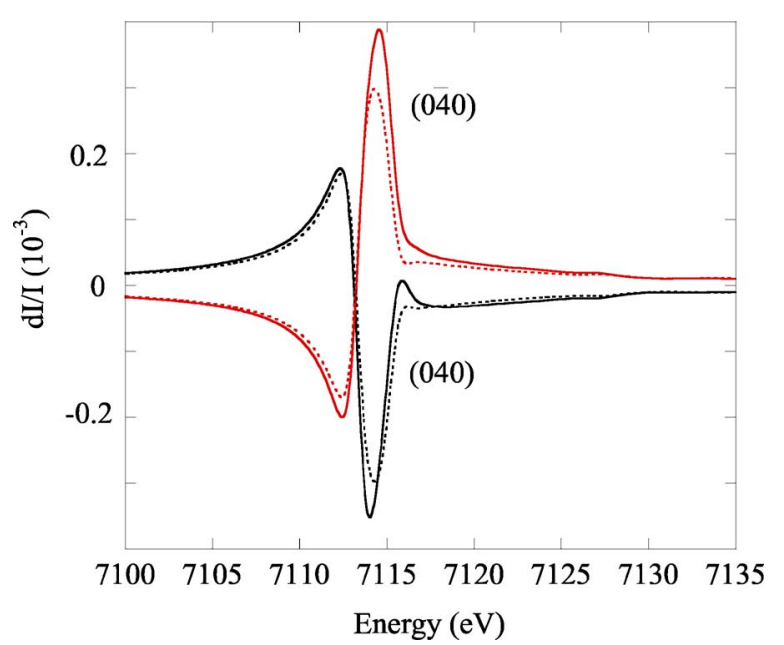

FIG. 3. (Color online) MEXS signal on a single set of equivalent iron sites $\left(\mathrm{Fe}_{1 a}\right)$. The total signal is shown as a solid line, while the dotted line shows the interference of the E1-E2 magnetic part with the simple Thomson term: in this case the inversion of the signal is exact. The origin of the difference between the two signals is explained in the text.

Finally, the analysis of the symmetry operations in Eq. (5) shows that only the following magnetic tensor components can be detected in the E1-E2 channel, when $k=$ even (the quantization axis coincides with the easy $c$ axis): $T_{1}^{(1)}-T_{-1}^{(1)}$ (toroidal moment), $T_{1}^{(2)}+T_{-1}^{(2)}$ (magnetic quadrupole), $T_{1}^{(3)}$ $-T_{-1}^{(3)}$ (toroidal octupole), and $T_{3}^{(3)}-T_{-3}^{(3)}$ (this latter cannot be detected in $\sigma \sigma$ polarization conditions and therefore is absent). The reason is that all tensors involved are both time reversal and parity odd, and therefore $\hat{m}_{z} T_{q}^{(p)}=(-)^{q+1} T_{q}^{(p)}$ and $\hat{T} \hat{m}_{x} T_{q}^{(p)}=(-)^{p} T_{-q}^{(p)}$. Given Eq. (5), the first rule selects, for $k=$ even, just the terms with $q=$ odd, whereas the second rule just picks up the four terms written above.

Compared to the interpretation of x-ray directional dichroism, ${ }^{8,12}$ the signal is determined by the same multipoles as the $c$-axis dichroism, which was also characterized

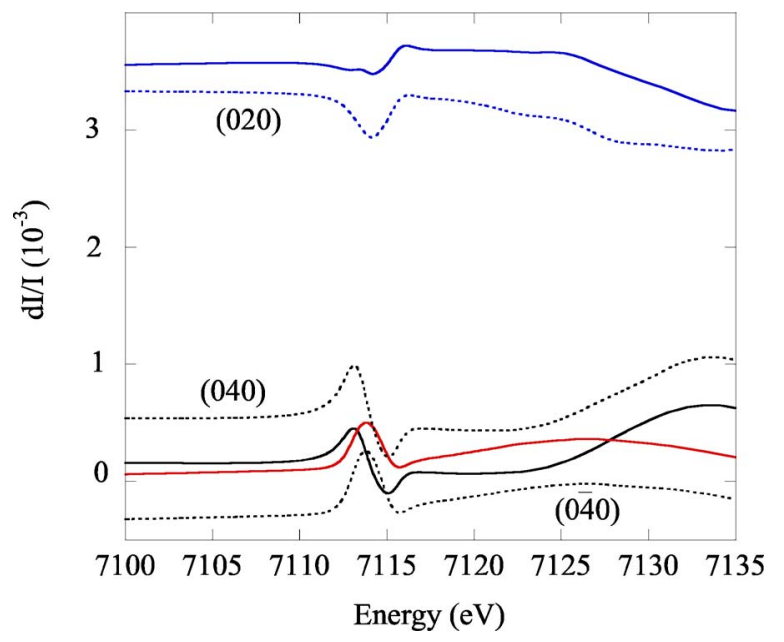

FIG. 4. (Color online) Comparison of the MEXS signal for two different fillings: the "nominal" one (dotted lines) and the real one (solid lines). by the absence of the $T_{3}^{(3)}-T_{-3}^{(3)}$ contribution: $:^{23}$ this could have been expected on the basis of the fact that the $\sigma \sigma$ polarization was chosen parallel to the $c$ axis. Moreover, as in that case, the lack of quantitative agreement for the $(0 \overline{4} 0)$ seems to imply that some of the terms involved in the scattering may be ascribed to correlation effects: these terms are not reproduced correctly by our independent particle approach, which, however, semiquantitatively describes many other aspects of the signal that depend only on the structure.

We end up the section by suggesting a method which, though rather complicated, could in principle allow us to single out each of the three multipoles experimentally. The azimuthal dependence of the three terms-toroidal dipole and octupole and magnetic quadrupole-is identical in the $\sigma \sigma$ channel, proportional to $\cos \phi$, where $\phi$ is the angle in the $a c$ crystallographic plane and $\phi=0$ when the polarization is along $c$. Therefore a single azimuthal scan is not useful to this aim. However, toroidal multipoles and magnetic quadrupole have different dependences when the outgoing polarization is neither orthogonal nor parallel to the scattering plane. If, for example, $\alpha$ is the angle in the plane orthogonal to the outgoing wave vector $\vec{q}^{\prime}$, measured from the $\sigma$ polarization, then

$$
T_{1}^{(1)}-T_{-1}^{(1)} \propto\left(\sin \phi \sin \theta_{B} \sin \alpha+\cos \phi \cos \alpha\right)
$$

and

$$
T_{1}^{(2)}+T_{-1}^{(2)} \propto\left(2 \sin \phi \sin \theta_{B} \sin \alpha-\cos \phi \cos \alpha\right) .
$$

The azimuthal dependence of the toroidal octupole is coincident with that of the toroidal moment. Therefore, toroidal multipoles and magnetic quadrupole could be, in principle, disentangled. However, through this procedure, new resonant magnetic terms could appear of $E 1-E 1$ origin, because of the rotated component of the polarization, which should be taken into account and subtracted from the signal.

From a theoretical point of view, the calculation through FDMNES is much simpler and gives an intensity ratio of the same order of magnitude for the three contributions. The energy dependence of each term is then shown in Fig. 5 for the (040) reflection.

\section{CONCLUSION}

In conclusion, by means of a numerical calculation through the FDMNES program we have confirmed the magnetic parity-breaking origin of the signal detected by means of an interference technique in resonant $\mathrm{x}$-ray scattering in Ref. 8. Such a signal shares several features in common with the analog one obtained through the nonreciprocal linear dichroism in Ref. 21 (and see also Ref. 12). However, the correct interpretation turns out to be slightly more involved due to the several aspects that play a role in a diffraction experiment, which we hope to have clearly illustrated with this paper.

Among the features shared with nonreciprocal linear dichroism, it may be interesting to underline the energy range of the signal. As already in the case of $\mathrm{V}_{2} \mathrm{O}_{3},{ }^{24}$ our numerical calculations confirm that magnetic E1-E2 signals are limited to the pre-edge region. 


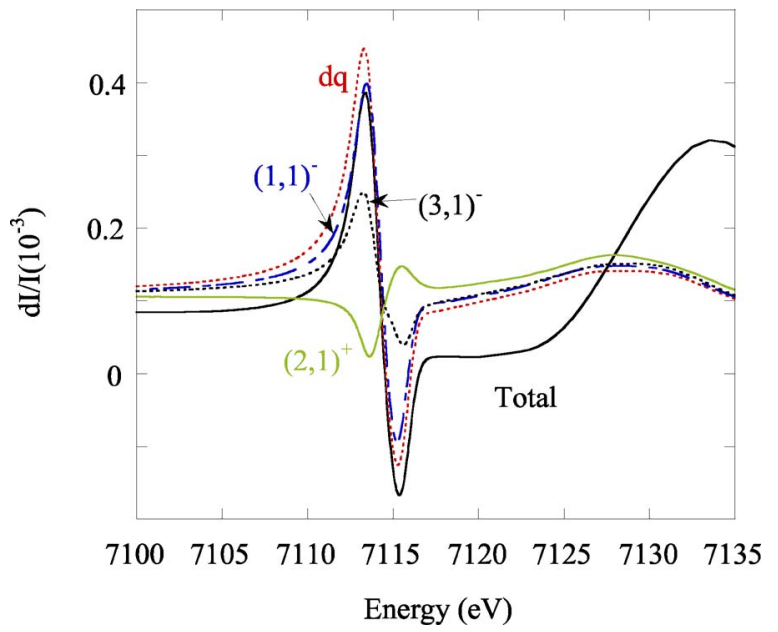

FIG. 5. (Color online) Tensor analysis of the MEXS signal, $T_{1}^{(1)}-T_{-1}^{(1)}, T_{1}^{(2)}+T_{-1}^{(2)}$, and $T_{1}^{(3)}-T_{-1}^{(3)}$, all E1-E2 contributions $(d q)$, and total signal (E1-E2 plus E2-E2).

Finally, we linger on two aspects of the MEXS that could be analyzed in the near future. The first is the disentanglement of the toroidal contribution from the magneticquadrupole one, as detailed already in the final part of the previous section. Such a disentanglement can be very important as it can tell us about the nature of higher-order magnetic distributions in this material, whether toroidal (i.e., corresponding to a circulation of magnetic moments around the resonant ion) or quadrupolar (i.e., corresponding to a configuration of two opposite magnetic moments around the resonant ion).

The second aspect is to detect, through MEXS, a primary order parameter of magnetoelectric origin. In Ref. 8, the temperature dependence of the MEXS signal was found to be coincident with the magnetization curve, thereby signaling that the MEXS order parameter is induced by the onset of the magnetic-dipole one. In this latter case, the induced order parameter is called secondary, whereas the magnetic dipole is the leading term in the expansion of Landau's free energy, and it is called primary order parameter. The possibility to have a phase transition driven by a higher-order magnetic multipole has been recently discovered for $\mathrm{NpO}_{2},{ }^{25}$ where a magnetic octupole order parameter with symmetry $\Gamma_{2}$ is commonly accepted to explain the low-temperature properties of the system. For what magnetoelectric order parameters are concerned, it would be worthwhile to investigate the possibility of such an effect in $\mathrm{Ti}_{2} \mathrm{O}_{3}$, a material that is known to be magnetoelectric, though nonmagnetic. ${ }^{26}$ Even though magnetoelectricity is usually attributed to higherorder terms in Landau's free energy, there is no definitive agreement in the literature on this point. The discovery of a system with a magnetic, space-inversion breaking primary order parameter would be a novelty in the field and could be found, in our opinion, only with the technique employed in Ref. 8 and described in the present article.

\section{APPENDIX A: WAVE-VECTOR DEPENDENCE OF E1-E2 SCATTERING}

The presence of the imaginary unit in the E1-E2 scattering amplitude [see Eqs. (2)-(4)] makes it out of phase with
TABLE III. Time-reversal properties of irreducible tensors in the E1-E2 channel.

\begin{tabular}{lccc}
\hline \hline & $\vec{\epsilon}_{i} \cdot \vec{\epsilon}_{o}$ & $\vec{\epsilon}_{i} \times \vec{\epsilon}_{o}$ & $\left\{\vec{\epsilon}_{i}, \vec{\epsilon}_{o}\right\}$ \\
\hline$\vec{q}_{i}-\vec{q}_{o}$ & $T^{(1)}$ & $\widetilde{T}^{(2)}$ & $T^{(1)}, T^{(2)}, T^{(3)}$ \\
$\vec{q}_{i}+\vec{q}_{o}$ & $\widetilde{T}^{(1)}$ & $T^{(2)}$ & $\widetilde{T}^{(1)}, \widetilde{T}^{(2)}, \widetilde{T}^{(3)}$ \\
\hline \hline
\end{tabular}

the $E 1-E 1$ and $E 2-E 2$ terms: for the latter nonmagnetic parts are real and magnetic parts are imaginary, while the opposite is true in the $E 1-E 2$ channel. In the framework of the classification given in Ref. 12 , it is possible to explicitly derive the polarization and wave-vector dependence in the E1-E2 approximation. In Table III we list the time-reversal properties of the allowed tensors, whether even or odd.

This table shows the way time-reversal odd (magnetic) $\widetilde{T}^{(p)}$ and time-reversal even (nonmagnetic) $T^{(p)}$ tensors of rank $p$ are coupled to the polarizations and wave vectors. The general tensor formalism can be recovered in Ref. 12 and references therein. Here we just want to show that in the $\sigma \sigma$ channel, where the second column does not contribute $\left(\epsilon_{i}\right.$ $\left.\times \epsilon_{o}=0\right)$, reversing just the wave vectors $\left(\vec{q}_{i} \rightarrow-\vec{q}_{o}\right.$ and $\vec{q}_{o}$ $\rightarrow-\vec{q}_{i}$ ) is equivalent to reversing the magnetization of the sample, because it is equivalent to the operation $\widetilde{T}^{(p)} \rightarrow$ $-\widetilde{T}^{(p)}$.

\section{APPENDIX B: DERIVATION OF EQ. (14)}

The following definitions are used:

$$
f_{0 k}+f_{0 k}^{O} \equiv u(k, p)+i v(k, p)
$$

with

$$
\begin{aligned}
u(k, p) \equiv & 4 \sum_{j=1}^{4}\left[p_{j} f_{a t}^{\mathrm{Fe}}+\left(1-p_{j}\right) f_{a t}^{\mathrm{Ga}}\right] \cos \left(2 \pi k y_{j}\right) \\
& +4 \sum_{j=1}^{6} f_{\mathrm{at}}^{\mathrm{O}} \cos \left(2 \pi k \tilde{y}_{j}\right), \\
v(k, p) \equiv & 4 \sum_{j=1}^{4}\left[p_{j} f_{a t}^{\mathrm{Fe}}+\left(1-p_{j}\right) f_{a t}^{\mathrm{Ga}}\right] \sin \left(2 \pi k y_{j}\right) \\
& +4 \sum_{j=1}^{6} f_{a t}^{\mathrm{O}} \sin \left(2 \pi k \tilde{y}_{j}\right), \\
f_{a}^{m} \equiv & {\left[f_{m}^{\prime}(k) \widetilde{u}(k, p)-f_{m}^{\prime \prime}(k) \widetilde{v}(k, p)\right] } \\
& +i\left[f_{m}^{\prime}(k) \widetilde{v}(k, p)+f_{m}^{\prime \prime}(k) \widetilde{u}(k, p)\right],
\end{aligned}
$$

with

$$
\tilde{u} \equiv 4 \sum_{j=1}^{4} p_{j} \cos \left(2 \pi k y_{j}\right)
$$




$$
\tilde{v} \equiv 4 \sum_{j=1}^{4} p_{j} \sin \left(2 \pi k y_{j}\right),
$$

and

$$
f_{a}^{n m} \equiv f_{n m 0}+f_{n m 2}
$$

where

$$
\begin{gathered}
f_{n m 0} \equiv\left[f_{n m 0}^{\prime} \widetilde{u}(k, p)-f_{n m 0}^{\prime \prime} \widetilde{v}(k, p)\right] \\
+i\left[f_{n m 0}^{\prime} \widetilde{v}(k, p)+f_{n m 0}^{\prime \prime} \widetilde{u}(k, p)\right] \\
f_{n m 2} \equiv 4 \sum_{j=1}^{4} p_{j}\left\{\left[f_{n m 2}^{\prime}(j) \cos \left(2 \pi k y_{j}\right)-f_{n m 2}^{\prime \prime}(j) \sin \left(2 \pi k y_{j}\right)\right]\right. \\
\left.+i\left[f_{n m 2}^{\prime \prime}(j) \cos \left(2 \pi k y_{j}\right)+f_{n m 2}^{\prime}(j) \sin \left(2 \pi k y_{j}\right)\right]\right\} \\
\equiv \operatorname{Re} f_{n m 2}(k)+i \operatorname{Im} f_{n m 2}(k)
\end{gathered}
$$

In the latter expressions, we have separated the scalar anomalous part $f_{n m 0}$, which does not depend on the site $j$, from the Templeton part $f_{n m 2}$, which does depend on the site $j$ and therefore cannot be extracted out of the sum. For the real and imaginary parts of $f_{n m 2}$ we have indicated the explicit $k$ dependence for later use. It is the latter term which is responsible for the difference between solid-line and dashedline signals in Fig. 3. Notice that in principle a similar dependence on $j$ holds also for the $f_{m}$ terms (it expresses the physical fact that toroidal moments may be different on the four unequivalent $\mathrm{Fe}$ ions), and it has been taken into account in the actual calculation through FDMNES: however, we have neglected it in this appendix in order not to make even more cumbersome an already heavy notation. In all the previous expressions we have underlined both the $\vec{Q}$ dependence (through $k$ ) and the dependence on the filling $p$. In particular, $f_{n m}^{\prime}$ and $f_{n m}^{\prime \prime}$ do not depend on $k$ and $f_{m}^{\prime}$ and $f_{m}^{\prime \prime}$ have an odd dependence on $k$. Therefore, one can write

$$
\begin{aligned}
d I= & {\left[f_{m}^{\prime}(k) \widetilde{u}(k, p)-f_{m}^{\prime \prime}(k) \widetilde{v}(k, p)\right] } \\
& \times[u(k, p) \\
& \left.+f_{n m 0}^{\prime} \widetilde{u}(k, p)-f_{n m 0}^{\prime \prime} \widetilde{v}(k, p)+\operatorname{Re} f_{n m 2}(k)\right]+\left[f_{m}^{\prime}(k) \widetilde{v}(k, p)\right. \\
& \left.+f_{m}^{\prime \prime}(k) \widetilde{u}(k, p)\right] \\
& \times\left[v(k, p)+f_{n m 0}^{\prime} \widetilde{v}(k, p)+f_{n m 0}^{\prime \prime} \widetilde{u}(k, p)+\operatorname{Im} f_{n m 2}(k)\right] \\
\text { and } & \\
d \widetilde{I}= & {\left[-f_{m}^{\prime}(k) \widetilde{u}(k, p)-f_{m}^{\prime \prime}(k) \widetilde{v}(k, p)\right] } \\
& \times\left[u(k, p)+f_{n m 0}^{\prime} \widetilde{u}(k, p)+f_{n m 0}^{\prime \prime} \widetilde{v}(k, p)+\operatorname{Re} f_{n m 2}(-k)\right] \\
& +\left[f_{m}^{\prime}(k) \widetilde{v}(k, p)-f_{m}^{\prime \prime}(k) \widetilde{u}(k, p)\right] \\
& \times\left[-v(k, p)-f_{n m 0}^{\prime} \widetilde{v}(k, p)+f_{n m 0}^{\prime \prime} \widetilde{u}(k, p)+\operatorname{Im} f_{n m 2}(-k)\right] .
\end{aligned}
$$

Summing them together and defining the terms involving $f_{n m 2}^{\prime}$ and $f_{n m 2}^{\prime \prime}$ as

$$
\begin{aligned}
& f_{n m 2}^{\prime} \equiv 4 \sum_{j=1}^{4} p_{j} f_{n m c}^{\prime}(j) \cos \left(2 \pi k y_{j}\right), \\
& f_{n m 2}^{\prime \prime} \equiv 4 \sum_{j=1}^{4} p_{j} f_{n m c}^{\prime \prime}(j) \cos \left(2 \pi k y_{j}\right), \\
& f_{n m 2}^{\prime} \equiv 4 \sum_{j=1}^{4} p_{j} f_{n m s}^{\prime}(j) \sin \left(2 \pi k y_{j}\right), \\
& f_{n m 2}^{\prime \prime} \equiv 4 \sum_{j=1}^{4} p_{j} f_{n m s}^{\prime \prime}(j) \sin \left(2 \pi k y_{j}\right),
\end{aligned}
$$

we recover Eq. (14).
${ }^{1}$ Y. Joly, S. Grenier, and J. E. Lorenzo, Phys. Rev. B 68, 104412 (2003).

${ }^{2}$ Y. Murakami, H. Kawada, H. Kawata, M. Tanaka, T. Arima, Y. Moritomo, and Y. Tokura, Phys. Rev. Lett. 80, 1932 (1998); Y. Murakami, J. P. Hill, D. Gibbs, M. Blume, I. Koyama, M. Tanaka, H. Kawata, T. Arima, Y. Tokura, K. Hirota, and Y. Endoh, ibid. 81, 582 (1998).

${ }^{3}$ S. B. Wilkins, P. D. Spencer, P. D. Hatton, S. P. Collins, M. D. Roper, D. Prabhakaran, and A. T. Boothroyd, Phys. Rev. Lett. 91, 167205 (2003).

${ }^{4}$ D. H. Templeton and L. K. Templeton, Acta Crystallogr., Sect. A: Cryst. Phys., Diffr., Theor. Gen. Crystallogr. 38, 62 (1982).

${ }^{5}$ V. E. Dmitrienko, Acta Crystallogr., Sect. A: Found. Crystallogr. 39, 29 (1983).

${ }^{6}$ R. Caciuffo, L. Paolasini, A. Sollier, P. Ghigna, E. Pavarini, J. van den Brink, and M. Altarelli, Phys. Rev. B 65, 174425 (2002).

${ }^{7}$ S. Di Matteo, Phys. Rev. B 70, 165115 (2005).
${ }^{8}$ Taka-hisa Arima, Jong-Hoon Jung, Masakazu Matsubara, Masato Kubota, Jin-Ping He, Yoshio Kaneko, and Yoshinori Tokura, J. Phys. Soc. Jpn. 74, 1419 (2005).

${ }^{9}$ Y. Murakami, JPSJ Online-News and Comments [March 13, 2005].

${ }^{10}$ Y. Joly, Phys. Rev. B 63, 125120 (2001). The program can be downloaded at the web address http:/wwwcristallo.grenoble.cnrs.fr/fdmnes

${ }^{11}$ T. Arima, D. Higashiyama, Y. Kaneko, J. P. He, T. Goto, S. Miyasaka, T. Kimura, K. Oikawa, T. Kamiyama, R. Kumai, and Y. Tokura, Phys. Rev. B 70, 064426 (2004).

${ }^{12}$ S. Di Matteo, Y. Joly, and C. R. Natoli, Phys. Rev. B 72, 144406 (2005).

${ }^{13}$ M. Blume, in Resonant Anomalous X-ray Scattering, edited by G. Materlik, J. Sparks, and K. Fisher (Elsevier, Amsterdam, 1994), p. 495. 
${ }^{14}$ In Eqs. (2) and (4), for the sign of the imaginary part, we have chosen the convention corresponding to the time dependence $e^{+i \omega t}$.

${ }^{15}$ The magnetic dipole term can be safely neglected in the x-ray range.

${ }^{16}$ The transition temperature depends on the crystal growth, as shown, e.g., in Ref. 11.

${ }^{17}$ J. P. Remeika, J. Appl. Phys. 31, 263S (1960).

${ }^{18}$ International Tables for Crystallography, 5th ed., edited by Theo Hahn (Kluwer, Dordrecht, 2002), No. 129.

${ }^{19}$ S. Di Matteo, Y. Joly, A. Bombardi, A. Paolasini, F. de Bergevin, and C. R. Natoli, Phys. Rev. Lett. 91, 257402 (2003).

${ }^{20}$ Notice that in Eqs. (12) and (13) only the E1-E1 contribution is present in $f_{a}^{n m}$, as E2-E2 terms are negligible in intensity and magnetic and nonmagnetic E1-E2 contributions are out of phase, so that $\operatorname{Re}\left[\left(f_{a}^{n m}\right)^{*} f_{a}^{m}\right]=0$ for $E 1-E 2$.

${ }^{21}$ M. Kubota, T. Arima, Y. Kaneko, J. P. He, X. Z. Yu, and Y. Tokura, Phys. Rev. Lett. 92, 137401 (2004).

${ }^{22}$ M. Blume and D. Gibbs, Phys. Rev. B 37, 1779 (1988).
${ }^{23}$ Notice, however, that in order to compare with the existing literature on nonreciprocal dichroism, in Ref. 12 a different choice of the quantization axis was considered (along $a$ instead of $c$ ).

${ }^{24}$ Y. Joly, S. Di Matteo, and C. R. Natoli, Phys. Rev. B 69, 224401 (2004).

${ }^{25}$ J. A. Paixao, C. Detlefs, M. J. Longfeld, R. Caciuffo, P. Santini, N. Bernhoeft, J. Rebizant, and G. H. Lander, Phys. Rev. Lett. 89, 187202 (2002); R. Caciuffo et al., J. Phys.: Condens. Matter 15, S2287 (2003).

${ }^{26}$ Magnetoelectricity in $\mathrm{Ti}_{2} \mathrm{O}_{3}$ is measured by D. N. Astrov, Zh. Eksp. Teor. Fiz. 38, 984 (1960). [Sov. Phys. JETP 11, 708 (1960)]. In S. C. Abrahams, Phys. Rev. 130, 2230 (1963), some antiferromagnetic neutron diffraction peaks were apparently found. However, subsequent neutron-polarized measurements showed that $\mathrm{Ti}_{2} \mathrm{O}_{3}$ is not magnetic in its low-temperature phase [see, e.g., H. Kendrick, A. Arrott, and S. A. Werner, J. Appl. Phys. 39, 585 (1968)]. 